\title{
Biomarker-based prediction of inflammatory bowel disease-related colorectal cancer: a case-control study
}

\author{
Monique M. Gerrits • Min Chen • Myrte Theeuwes - Herman van Dekken • \\ Marjolein Sikkema • Ewout W. Steyerberg • Hester F. Lingsma • Peter D. Siersema • \\ Bing Xia • Johannes G. Kusters • C. Janneke van der Woude • Ernst J. Kuipers
}

Accepted: 10 November 2010 / Published online: 17 February 2011

(C) The Author(s) 2011. This article is published with open access at Springerlink.com

\begin{abstract}
Background Regular colonoscopic surveillance for detection of dysplasia is recommended in longstanding inflammatory bowel disease (IBD), however, its sensitivity is disputed. Screening accuracy may increase by using a biomarker-based surveillance strategy.

Methods A case-control study was performed to determine the prognostic value of DNA ploidy and p53 in IBD-related neoplasia. Cases with IBD-related colorectal cancer (CRC), detected in our surveillance program between 1985-2008, were selected and matched with two controls, for age, gender, disease characteristics, interval of follow-up, PSC,
\end{abstract}

Monique M. Gerrits and Min Chen contributed equally to this work.

M. M. Gerrits $\cdot$ M. Chen $\cdot$ M. Theeuwes $\cdot$ M. Sikkema $\cdot$

P. D. Siersema · J. G. Kusters · C. J. van der Woude · E. J. Kuipers

Department of Gastroenterology and Hepatology,

Erasmus MC - University Medical Center,

Rotterdam, The Netherlands

M. Chen

Department of Geriatrics, Zhongnan Hospital, Wuhan University,

Wuhan, China

H. van Dekken

Department of Pathology,

Erasmus MC - University Medical Center,

Rotterdam, The Netherlands

E. W. Steyerberg $\cdot$ H. F. Lingsma

Department of Public Health,

Erasmus MC - University Medical Center,

Rotterdam, The Netherlands

\section{B. Xia}

Department of Gastroenterology and

Department of Internal Medical, Zhongnan Hospital,

Wuhan University School of Medicine,

Wuhan, China and previous surgery. Biopsies were assessed for DNA ploidy, p53, grade of inflammation and neoplasia. Progression to neoplasia was analyzed with Cox regression analysis, adjusting for potentially confounding variables.

Results Adjusting for age, we found statistically significant Hazard ratios (HR) between development of CRC, and low grade dysplasia (HR5.5; 95\%CI 2.6-11.5), abnormal DNA ploidy (DNA index (DI) 1.06-1.34, HR4.7; 95\%CI 2.9-7.8 and DI $>1.34$, HR6.6; 95\%CI 3.7-11.7) and p53 immunopositivity (HR3.0; 95\%CI 1.9-4.7) over time. When adjusting for all confounders, abnormal DNA ploidy (DI 1.06-1.34, HR4.7; 95\%CI 2.7-7.9 and DI $>1.34$, HR5.0; 95\%CI 2.5-10.0)

M. M. Gerrits $(\varangle)$

Department of Clinical Genetics,

Maastricht University Medical Centre,

P.O. box 5800, P. Debyelaan 25,

6202 AZ Maastricht, The Netherlands

e-mail: monique.gerrits@mumc.nl

Present Address:

P. D. Siersema

Department of Gastroenterology and Hepatology,

University Medical Center Utrecht,

Utrecht, The Netherlands

Present Address:

J. G. Kusters

Department of Medical Microbiology,

University Medical Center Utrecht,

Utrecht, The Netherlands 
and p53 immunopositivity (HR1.7; 95\%CI 1.0-3.1) remained statistically significant predictive of neoplasia.

Conclusion In longstanding IBD, abnormal DNA ploidy and p53 immunopositivity are important risk factors of developing CRC. The yield of surveillance may potentially increase by adding these biomarkers to the routine assessment of biopsies.

Keywords Inflammatory bowel disease $\cdot$ Colorectal cancer S Surveillance $\cdot$ Abnormal DNA ploidy $\cdot$ p53 immunopositivity

\section{Introduction}

Inflammatory bowel disease (IBD), which includes ulcerative colitis (UC) and Crohn's disease (CD), is a chronic inflammatory disorder of the intestine. With approximately 4 million patients affected in the Western world, IBD is one of the most common chronic inflammatory disorders. Epidemiological evidence indicates that patients with IBD are at increased risk of developing colorectal cancer (CRC). The risk is considerable as it has been estimated that up to one in five patients will develop CRC within 30 years after an initial diagnosis of IBD [9].

Duration of the disease is a major clinical risk factor for IBD-related CRC, with a relative risk with long-standing colitis increasing to 6-18 [6, 7]. Most IBD-related CRC is seen in patients with pancolitis, whereas proctitis and leftsided colitis are associated with a small to intermediate cancer risk. Other risk factors for IBD-related CRC include young age at onset of IBD, presence of primary sclerosing cholangitis, presence of pseudopolyps, and a positive family history for CRC $[1,6,7,19]$.

Current guidelines from the American Gastroenterology Association (AGA) [2] and the British Society of Gastroenterology (BSG) $[4,8]$ recommend colonoscopic surveillance every 1 to 5 years for IBD-colitis patients starting 8 to 10 years after the initial diagnosis. This surveillance aims at early detection of neoplastic lesions, at a treatable stage [6]. Detection of dysplasia is generally accepted as the gold standard to predict cancer risk. Dysplasia as risk predictor for IBD-related cancer is however known to have significant shortcomings.

Dysplasia, which is commonly sub classified into two distinct morphological stages, i.e., low-grade dysplasia (LGD) and high-grade dysplasia (HGD), often arises multifocally throughout the colon of IBD patients. As dysplastic lesions are difficult to recognize by conventional endoscopy, the obtained biopsy specimens are prone to sampling error $[18,33]$. In addition, misinterpretation of dysplasia occurs due to inter- and intraobserver variability between pathologists, and by the co-existent presence of variable degrees of inflammation $[9,26,33]$.
In the search of better markers for the prediction of IBDrelated CRC development, a variety of biomarkers have been studied, including DNA ploidy, and mutations of the $A P C$ gene, K-ras gene, DCC gene and p53 tumor suppressor gene $[5,18]$. Of these, DNA ploidy and $p 53$ gene mutations currently seem to be the most promising candidates $[5,16,18,25,34]$. Both markers are observed early during the cascade of CRC development and, in addition, they are assumed to be diffusely expressed thus decreasing the risk of sampling error [21, 22].

As the relative risk of abnormal DNA ploidy and p53 immunopositivity in developing IBD-related CRC is currently unknown, we investigated the prognostic value of these markers for neoplastic progression in a case-control study design by testing surveillance biopsies of high-risk IBD patients (biopsy specimens taken $\geq 8$ years after the diagnosis of IBD), who either or not progressed towards CRC.

\section{Materials and methods}

\subsection{Patients selection}

This retrospective case-control study was conducted in the IBD patient cohort of the Erasmus MC, Rotterdam, the Netherlands. Cases were high-risk IBD patients with documented neoplastic progression (defined as patients with progression to $\mathrm{CRC}$ ). Each case was preferentially matched with two controls. Controls were IBD-colitis patients without signs of neoplastic progression (defined as patients without HGD or CRC) during surveillance. Cases and controls were matched for age (with a maximum of 2 years difference), gender, colitis characteristics (i.e. extension and underlying $\mathrm{UC}$ or $\mathrm{CD}$ ), age at onset of colitis symptoms, disease duration, interval of follow-up since the first colonoscopy (with a maximum of 1 year difference), presence of PSC, and previous surgery. Inclusion criteria for cases were (i) IBD history $\geq 8$ years; (ii) confirmed IBD by colonoscopy and histology; and (iii) having undergone at least one surveillance colonoscopy with biopsy sampling prior to the development of neoplasia. An exclusion criterion was IBD with dysplasia or CRC at the first colonoscopy.

Biopsies specimens collected at routine surveillance colonoscopies or colectomy between 1985 and 2008 in our institute were retrieved and analyzed for histology, DNA ploidy and p53 as described below. They consist of 1,671 samples obtained from different locations of the colon and originated from 54 unrelated patients. Use of patient material was approved by the Medical Ethical Committee of the Erasmus MC.

Since 2001, a standardized biopsy protocol was used (four-quadrant biopsy specimens every $10 \mathrm{~cm}$ ). Before 2001, the biopsy protocol was not standardized. 


\subsection{Histology}

Formalin-fixed paraffin-embedded tissue samples were serially sectioned at 4 or $50 \mu \mathrm{m}$. The first and last section $4 \mu \mathrm{m}$ were stained with hematoxylin \& eosin stain and light microscopically evaluated. The degree of inflammation and dysplasia grade or presence of CRC was reevaluated by one expert gastrointestinal pathologist without knowledge of clinical and biomarker status, using the Geboes scoring system [10] and the International Classification of Dysplasia in Inflammatory Bowel Disease [29]. Patients were classified according to the most severe abnormality present in the biopsy specimens.

\subsection{DNA ploidy by flow cytometry}

DNA ploidy analysis was performed on formalin-fixed paraffin-embedded biopsy specimens using a pepsinization technique, which was modified from Hedley et al. [15]. For each sample, one $50-\mu \mathrm{m}$-thick section was obtained from the paraffin block. Following deparaffinization in xylene and subsequent rehydration. The tissue was suspended in phosphate-buffered-saline (PBS). Then, the specimens were incubated in $0.05 \%$ protease (Sigma-Aldrich, Zwijdrecht, The Netherlands) for $45 \mathrm{~min}$ at $37^{\circ} \mathrm{C}$, minced mechanically with a syringe and a $60-\mu \mathrm{m}$ needle, and filtered through a $50-\mu \mathrm{m}$ nylon mesh. Subsequently, the suspension was centrifuged for $10 \mathrm{~min}$ at $900 \mathrm{~g}$, the supernatant was discarded and the pellet was resuspended in PBS containing 0.01\% RNAse (Simga-Aldrich). For DNA staining, 0.01\% propidium iodide was added to the samples. At least 10,000-15,000 nuclei per specimen were analyzed by FACScan flow cytometry (Becton Dickinson, San Jose, CA). Data analysis was performed using CellQuest (Becton Dickinson) and Modfit 3.1 software packages (Verity Software House, Inc, Topsham, ME, USA). The DNA index (DI) was calculated as the ratio of the abnormal $\mathrm{G}_{0} / \mathrm{G}_{1}$ mean peak channel number to the normal diploid $G_{0} / G_{1}$ mean peak channel number. Histograms displaying a $\mathrm{G}_{0} / \mathrm{G}_{1}$ peak with a coefficient of variation (CV) of $1 \% \leq \mathrm{CV} \leq 10 \%$ were included in the analysis. Samples were considered diploid when the DNA index was $0.95 \leq \mathrm{DI} \leq 1.05$, near diploid in case of $1.06 \leq \mathrm{DI} \leq 1.34$, and aneuploid in case of a DI $>1.34$ [11, 32]. DNA histograms were classified without knowledge of the histological diagnosis.

\section{4 p53 immunohistochemistry}

Immunohistochemistry for p53 was performed on $4 \mu \mathrm{m}$ formalin-fixed paraffin-embedded tissue slide. After deparaffinization in xylene and rehydration through graded ethanol, the sections were incubated with $3 \% \mathrm{H}_{2} \mathrm{O}_{2}$ in methanol for $10 \mathrm{~min}$. Then, the section were microwaved in monocitric acid buffer pH 6.0 for 20 min, washed, and blocked with $10 \%$ normal human plasma, 10\% goat serum (Dakocytomation, Heverlee, Belgium) and $5 \%$ bovine serum albumin (Sigma-Aldrich) in Tris- $\mathrm{HCl}$ pH 9.0. p53 immunostaining was accomplished using a mouse monoclonal antibody, i.e. clone DO-7 (1:100, Dako-cytomation). After overnight incubation with DO-7 at $4{ }^{\circ} \mathrm{C}, \mathrm{p} 53$ signals were amplified with Envision (Dako-cytomation). Subsequently, 3'-3'-diaminobenzidine ( $1 \mathrm{~g} / \mathrm{L}$, Sigma-Aldrich) was added to the slide to detect $\mathrm{p} 53$. The slides were counterstained in Harris' hematoxylin, dehydrated and mounted. To determine the intraobserver variability between the different experiments, control slides $(n=3)$ of tissue that previously had shown to express p53 were included in the experiments. When the results of control slides differed from the results of previous staining, data were excluded from the analysis and the experiment was repeated. Substitution of the primary antibody by $\mathrm{IgGb} 2$ (Dako-cytomation) or Tris- $\mathrm{HCl} \mathrm{pH} 9.0$ in a matched serial section was used as a negative control. The intensity and percentage of positive cells were evaluated by two experts without knowledge of clinical and DNA ploidy status. Overexpression of p53 was defined as moderate and intense brown staining in $>15 \%$ of the nuclei [14].

\subsection{Statistical analysis}

Statistical analyses were conducted using SPSS software (SPSS version 16.0, Chicago, Illinois, USA) and $\mathrm{R}$ software ( $\mathrm{R}$ foundation for Statistical Computing, version 2.6.2).

Median and 25-75 percentiles were calculated for all continuous variables while proportions were calculated for all categorical variables.

At each follow-up visit (colonoscopies performed on regular basis $\geq 8$ years after the diagnosis of IBD), histology, expression of the biomarkers, and grade of inflammation were determined in biopsy specimens taken from different locations of the colon of the same patient. For the analysis, we used from each time point the biopsy that was most different from normal (e.g. if for one patient at one time point one biopsy was aneuploid, one near diploid and three diploid, that time point was scored aneuploid).

To estimate the predictive value of histology, DNA ploidy, p53 and grade of inflammation we used Cox proportional hazard regression with time dependent covariates to take the multiple time points per patient into account. The relationship between these predictors and IBD-related neoplasia was expressed in hazard ratios (HR) with 95\% confidence intervals (CI). We first adjusted for age, then for age and LGD and finally for the complete set of confounders (age, LGD, DNA ploidy, p53 immunopositivity and grade of inflammation). 
Random effect regression analysis was used to calculate the statistical significance level of the correlation between outcome and the different predictors. This model takes the dependency between biopsy specimens per follow-up visit per patient into account. The data is presented by grade of neoplasia. $P$-values $<0.05$ were considered statistically significant. Smoothing splines were used to visualize trends over time for the biomarkers, DNA ploidy and p53 immunopositivity.

\section{Results}

\subsection{Patient characteristics}

In our cohort of IBD patients who underwent colonoscopic surveillance at the Erasmus MC-Rotterdam between 1985 and 2008, 36 patients developed CRC. Sixteen patients were excluded from the study because of an IBD history $<8$ years, HGD or CRC at their first surveillance colonoscopy, or lack of surveillance biopsies. For the twenty remaining IBD cases
(CD, $n=8$; UC, $n=12$ ), 844 biopsy specimens, with a median (25-75 percentiles) of $6.0(4.0-10.0)$ biopsies per colonoscopy or colectomy, were analyzed. 730 biopsy specimens had been taken prior to the development of HGD and/or CRC, and 171 specimens at the time of HGD and/or CRC. Cases were matched with 34 controls (CD, $n=14$; UC, $n=$ 20). From these, in total 827 biopsy specimens obtained during colonoscopic surveillance or colectomy were included. For six cases (CD, $n=2$; UC, $n=4)$, no second matched control fulfilling all selection criteria could be selected from our patient cohort.

All patients' characteristics are given in Table 1. No significant differences were observed between the cases and controls in age, gender, disease characteristics, interval of follow-up, treatment during surveillance, family history, PSC, and previous surgery.

During surveillance, ten colectomies were recommended for reasons of dysplasia (all cases), and six as a result of severe colitis (cases, $n=2$; controls, $n=4$ ). One patient with LGD refused to undergo surgery. In seven of the nine colectomies after a previous biopsy-based diagnosis of

Table 1 Characteristics of inflammatory bowel disease (IBD) patients

\begin{tabular}{|c|c|c|c|}
\hline Variables & Cases $(n=20$ patients $)$ & Controls ( $n=34$ patients) & $p$-value \\
\hline Age at IBD diagnosis, $y$ & $25.3(19.2-38.4)$ & $25.8(18.9-34.8)$ & 0.6 \\
\hline Male, $n$ & $20(75.0 \%)$ & $24(70.6 \%)$ & 0.7 \\
\hline IBD history, $y$ & $17.0(12.0-23.8)$ & $18.5(14.0-23.3)$ & 0.7 \\
\hline Follow-up period, $y$ & $8.0(1.3-11.5)$ & $7.2(2.2-12.6)$ & 0.8 \\
\hline Colonoscopies/follow-up period, $n$ & $4.4(1.5-7.0)^{\mathrm{a}}$ & $4.3(2.0-5.3)$ & 0.8 \\
\hline Interval between follow-up colonoscopies, $y$ & $1.6(0.6-2.3)^{\mathrm{a}}$ & $2.0(1.0-2.4)$ & 0.04 \\
\hline Total number of follow-up visits & 116 & 148 & - \\
\hline Type of colitis, $n$ & & & 0.2 \\
\hline Proctitis & $3(15.0 \%)$ & $1(2.9 \%)$ & \\
\hline Left-sided colitis & $3(15.0 \%)$ & $12(35.3 \%)$ & \\
\hline Pancolitis & $14(70.0 \%)$ & $20(58.8 \%)$ & \\
\hline Right-sided colitis & - & $1(2.9 \%)$ & \\
\hline Positive family history, $n$ & $2(10.0 \%)$ & $4 / 34(11.8)$ & 0.8 \\
\hline Primary sclerosing cholangitis, $n$ & $6(30.0 \%)$ & $6(17.6 \%)$ & 0.3 \\
\hline Medication use & $18(90.0 \%)^{\mathrm{b}}$ & $34(100 \%)$ & \\
\hline Aminosalicylates, $n$ & $15(83 \%)$ & $31(91 \%)$ & 0.4 \\
\hline Corticosteroids, $n$ & $11(61 \%)$ & $21(62 \%)$ & 1.0 \\
\hline Immunosuppressives, $n$ & $7(38 \%)$ & $11(32 \%)$ & 0.6 \\
\hline Biologicals, $n$ & $2(11 \%)$ & $2(6 \%)$ & 0.5 \\
\hline Surgery during surveillance, $n$ & $5(25.0 \%)$ & $4(11.4 \%)$ & 0.2 \\
\hline Total number of biopsies, $n$ & 844 & 827 & - \\
\hline Biopsies per colonoscopy & $6.0(4.0-10.0)$ & $5.6(3.0-7.0)$ & 0.08 \\
\hline
\end{tabular}

${ }^{\text {a }}$ Prior to the development HGD and/or CRC

${ }^{\mathrm{b}}$ Information on medication use of two patients was missing

$y$, years; $n$, number

Median (25-75 percentiles) given for continuous variables 
dysplasia, HGD and/or CRC lesions were detected by the pathologist. No sign of neoplasia were seen in the colectomies of the patients with severe colitis. All patients without histological conformation of HGD or CRC continued the surveillance program.

\subsection{Histology}

Grading surveillance biopsies according to the International Classification of Dysplasia in Inflammatory Bowel Disease [29] revealed that the majority of specimens (1123/1671; $67.2 \%$ ) exhibited no dysplasia (ND). Biopsy specimens of one follow-up visit of the cases could not be graded due to severe architectural changes. When the specimens were analyzed per follow-up visit (Fig. 1a), ND was seen in $53.0 \%(61 / 115)$ of the follow-up visits in the cases, compared to $87.8 \%(130 / 148)$ in the controls. Indefinite dysplasia (IND) was found in 16.5\% (19/115) and $11.5 \%$
(17/148), respectively. LGD was detected in $7.8 \%(9 / 115)$ of the follow-up visits in the cases, compared to $0.7 \%(1 / 148)$ in the controls. HGD and CRC were detected in 5.2\% $(6 / 115)$ and $17.4 \%$ (20/115) of the follow-up visits in cases, compared to none in controls.

By endoscopy and macroscopy examination of the colon after colectomy, 80\% (29/36) of the abnormal lesions were seen. Seventeen lesions were raised. Four lesions with dysplasia (LGD $n=3, \mathrm{HGD}=1$ ) were missed by the endoscopists and were only detected histologically. For three lesions, the data on morphology of the lesion was missing.

When comparing the different specimens obtained from a single patient at a single time point, we found that $41.6 \%(14 /$ 36) of the sample-sets displayed differences in the grading between the individual samples from that particular time point. In most of these cases $(n=9), \mathrm{HGD}$ and CRC at one spot was accompanied by LGD or HGD at another spot. At five time points, biopsy specimens with neoplasia were
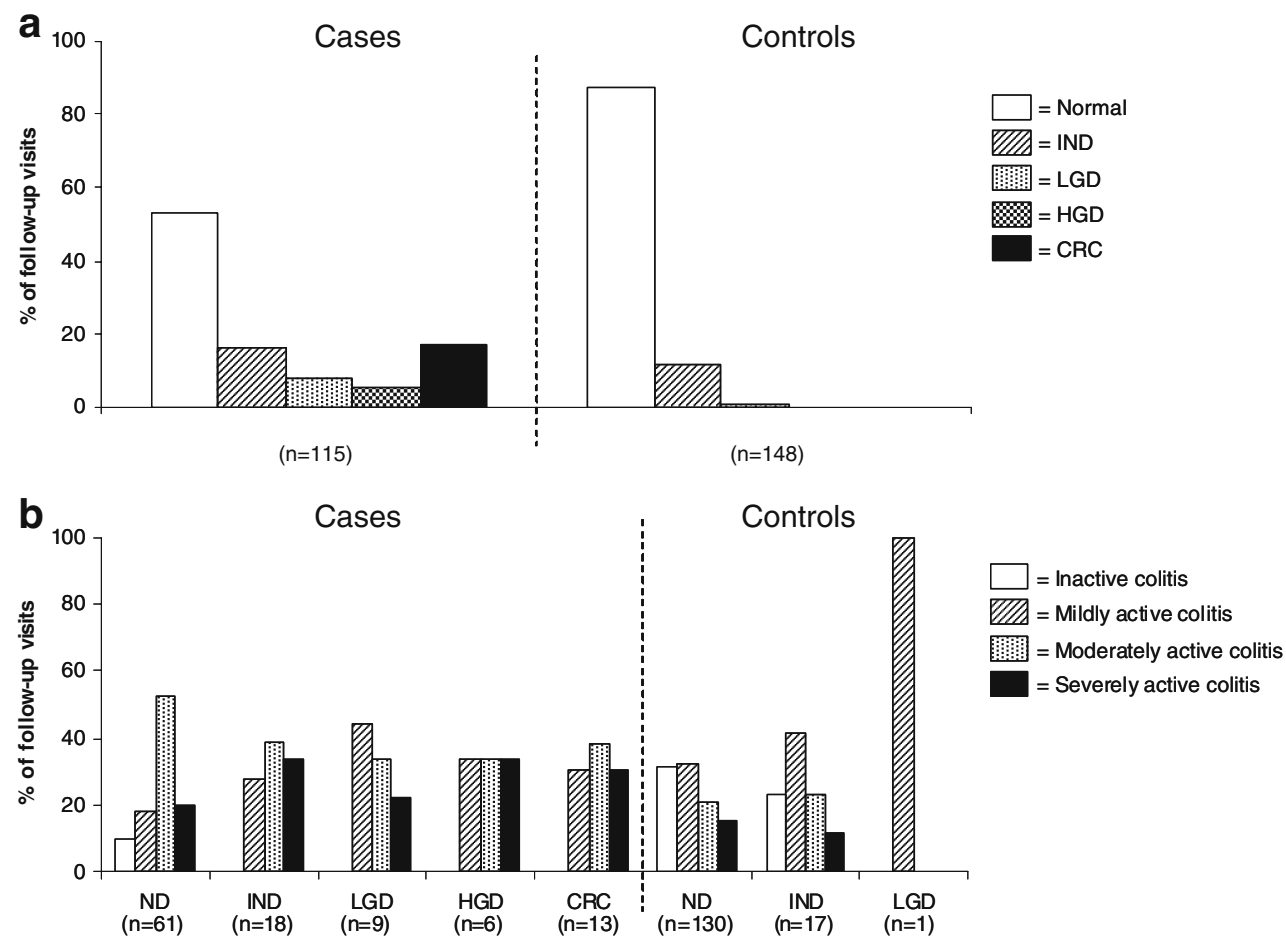

Fig. 1 Prevalence of neoplasia and inflammatory activity in different IBD subgroups. a Percentages of follow-up visits with neoplasia graded according to the International Classification of Dysplasia in Inflammatory Bowel Disease [29] in the case and control group. Biopsy specimens of one follow-up visit of the cases could not be graded due to severe architectural changes. $P$-value from random effect regression analyses for difference between the cases and controls in grade of neoplasia is $p=0.7$ for IND, $p=0.8$ for LGD, $p=1.00$ for HGD and $p=1.00$. In the ND-group this analysis revealed $p$-value of $p=0.8$ for IND. ND has been used as reference conditions. b Inflammatory activity graded according to the Geboes scoring system [10] in different subgroups of IBD patients. Biopsy specimens of eight follow-up visits of the cases could not be graded due to severe architectural changes or limited amount of tissue (IND $n=1$, CRC $n=$ 7). $P$-value from random effect regression analyses for difference between the cases and controls in inflammatory activity are $p=0.8$ for mild colitis, $p=0.7$ for moderate colitis and $p=0.7$ for severe colitis. In the ND-group the same analysis revealed $p$-values of $p=0.9, p=0.7$ and $p=0.8$, respectively. Inactive colitis has been used as reference conditions. Normal, $N D$, no dysplasia; $I N D$, indefinite dysplasia; $L G D$, low grade dysplasia; $H G D$, high grade dysplasia; $C R C$, colorectal cancer (CRC). Mild colitis, neutrophil infiltration of $<50 \%$ with no crypt destruction, erosion or ulceration; moderate colitis, neutrophil infiltration of $>50 \%$ with no erosion or ulceration; severe colitis, erosion or ulceration 
found together with biopsy specimens without neoplasia (no LGD, HGD and CRC). As 66\% (10/15) the colonoscopies were performed before the standardized biopsy protocol of 2001 , data on the exact location of the biopsy is scarce.

We also graded the surveillance biopsies according to the Geboes scoring system [10]. Biopsy specimens of eight follow-up visits of the cases could not be graded due to severe architectural changes or limited amount of tissue (IND $n=1$, CRC $n=7$ ). Moderate colitis was more often seen in cases per follow-up visit than in controls, even before LGD, HGD and/or CRC occurred (Fig. 1b).

When comparing the sample-sets from a single patient at a single time point with neoplasia, differences in inflammatory activity between the individual samples was observed $16.6 \%(6 / 36)$ of the sample-sets. The highest inflammatory activity was always observed in samples obtained at the location with most severe histological abnormality of that particular time point.

\subsection{DNA ploidy}

Biopsy specimens of four follow-up visits (ND $n=3$, LGD $n=1)$ of the cases and fourteen follow-up visits of the controls (ND $n=10$, IND $n=4$ ) could not be graded due to severity of inflammation or a limited amount of tissue. DNA aneuploidy (DI >1.34) in the cases was detected in $28.2 \%(33 / 110)$ of the follow-up visits. A near-diploid (DI 1.06 to 1.34 ) and diploid (DI 0.96 to 1.05 ) DNA content was seen respectively in $46.4 \%(51 / 110)$ and $25.5 \%(28 /$ $110)$ of the follow-up visits. For the controls, DNA aneuploidy was found in $2.2 \%$ (3/134) of the follow-up visits, and a near-diploid and diploid DNA content was observed in $3.0 \%(4 / 134)$ and $94.8 \%$ (127/134) of the follow-up visits, respectively.

When the cases were subdivided by grade of neoplasia, DNA aneuploidy was found in 50\% (10/20) of the followup visits with CRC versus $16.7 \%(1 / 6)$ in those with HGD, $62.5 \%(5 / 8)$ in those with LGD, $31.6 \%(6 / 19)$ in those with IND, and $17.2 \%(10 / 58)$ in those with ND, while a neardiploid DNA content was seen in 45.0\% (9/20), 33.3\% (2/6), $12.5 \%(1 / 8), 52.6 \%(10 / 19)$, and $50.0 \%$ (29/58), respectively (Fig. 2a).

In $27.7 \%$ (10/36) sample-sets obtained from single patients with neoplasia at a particular time point, differences in DI were seen between the individual biopsy specimens. The DI was the highest at the most severe histological abnormality present at that particular time point. In the sample-sets, where biopsy specimens with HGD and/or CRC were found together with specimens without neoplasia ( $n=5,3$ sporadic lesions, 2 multifocal lesions), an abnormal DNA content was detected in specimens without neoplasia at a maximal distance of $65 \mathrm{~cm}$ from the lesion.
Five years prior to the development of $\mathrm{CRC}$, an increase in the proportion of follow-up visits with DNA aneuploidy was found for the cases from 18 to $43 \%$. In general, once DNA ploidy was positive it stayed positive over time. When a DI $\geq 1.06$ was used as determinant, already $\sim 70 \%$ of cases were positive 10 years prior to the ultimate diagnosis of CRC, and this proportion remained stable over time. In the controls, the values were $<5 \%$, and no increase in the proportion of followup visits with an abnormal DNA content was seen over time (Fig. 3a).

\section{4 p53 expression}

Biopsy specimens of four follow-up visits of the cases (ND $n=$ 3 , CRC $n=1$ ) and ten follow-up visits of controls (ND $n=9$, IND $n=1$ ) could not be graded due to severity of inflammation or a limited amount of tissue. p53 immunopositivity in the cases was found in $37.3 \%$ (41/110) of the follow-up visits (Fig. 2b). By grade of neoplasia, these values were $73.7 \%$ (14/19) of the follow-up visits with CRC, $66.7 \%$ (4/6) with HGD, 55.6\% (5/9) with LGD, 26.3\% (5/19) with IND, and $24.1 \%(14 / 58)$ with ND. For the controls, only $2.9 \%(4 / 138$; IND $1 / 16$ and ND 3/121) of the follow-up visits were $\mathrm{p} 53$ positive.

In $16.6 \%(6 / 36)$ of the sample-sets obtained from single patients with neoplasia at a particular time point, differences in p53 immunopositivity were seen between the different biopsy specimens. p53 immunopositivity was found at the most severe histological abnormality present at that particular time point. In the sample-sets, where biopsy specimens with HGD and/or CRC were found together with specimens without neoplasia $(n=5)$, p53 immunopositivity was detected in specimens without neoplasia at a maximal distance of $55 \mathrm{~cm}$ from the lesion.

Prior to the development of $\mathrm{CRC}$, an increase in the proportion of follow-up visits with p53 immunopositivity was seen in the cases from 10 to $75 \%$. Once p53 was positive it remained positive over time. The increase in p53 immunopositivity was predominant at the time close to the development of CRC. In controls, p53 immunopositivity was detected in $<5 \%$, and no increase in proportion was detected over time (Fig. 3b).

When the p53 status of the cases was combined with the DNA ploidy data, only $14.8 \%(16 / 108)$ of the followup visits were p53 positive and had a DI $>1.34$ (aneuploid), in ten different patients, compared to none of the controls (Fig. 2c). When DNA ploidy with a DI of 1.06 to 1.34 (near-diploid) was considered as positive, $13.8 \%(13 / 108)$ of the follow-up visits in the cases were positive for both markers, in eight different patients, compared to one $(0.8 \%, 1 / 130)$ follow-up visit of one control patient. 

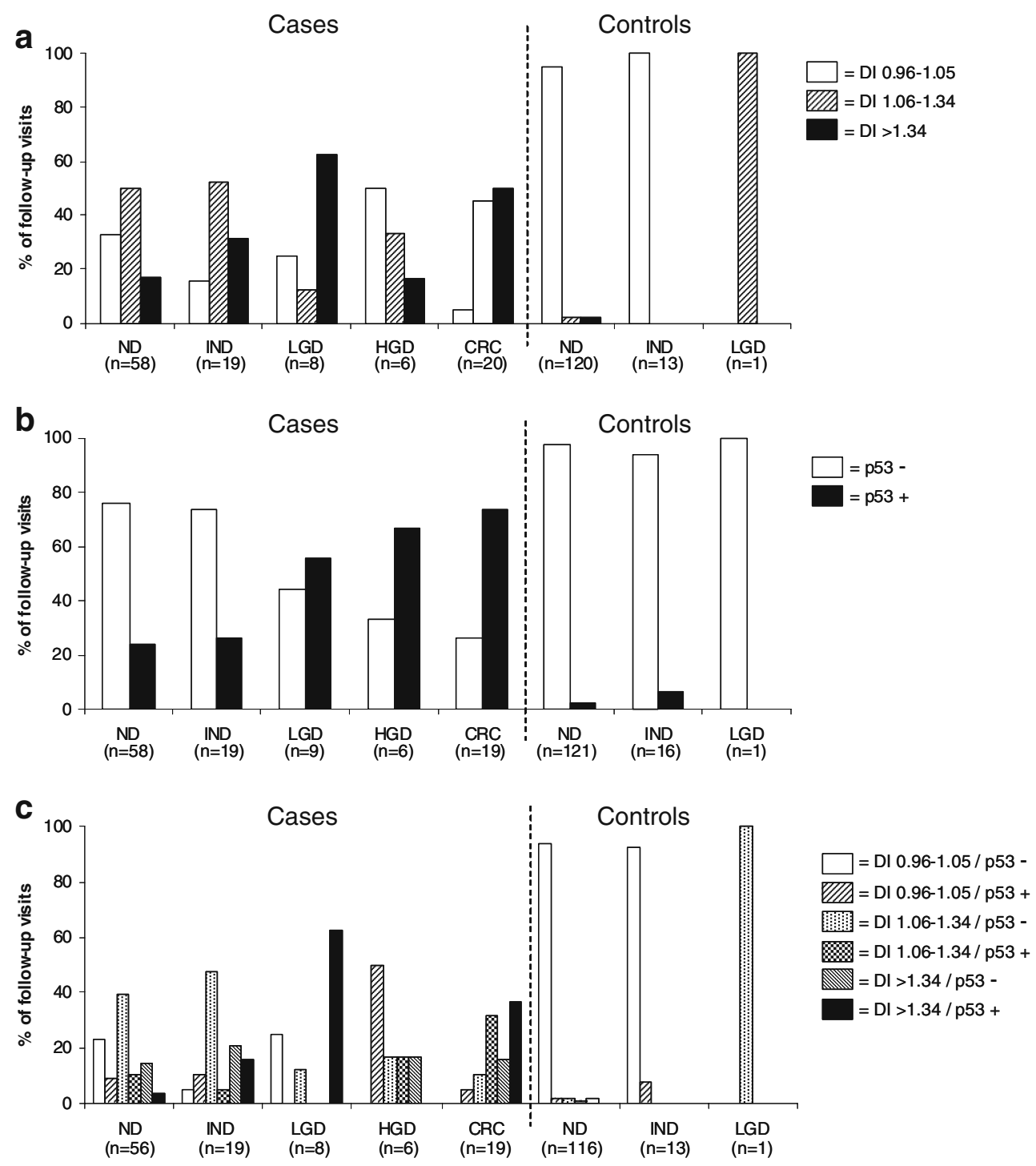

Fig. 2 Prevalence of abnormal DNA content and p53 immunopositivity in different IBD subgroups. a Percentages of abnormal DNA content determined by flow cytometry in different subgroups of IBD patients. Biopsy specimens of four follow-up visits (ND $n=3$, LGD $n=1$ ) of the cases and fourteen follow-up visits of the controls (ND $n=10$, IND $n=4$ ) could not be graded due to severity of inflammation or a limited amount of tissue. $P$-values from random effect regression analyses for difference between the cases and controls in abnormal DNA content are $p=0.009$ for DI $1.06-1.34$ and $p=0.0 .07$ for DI $>1.35$. In the ND-group the same analysis revealed $p$-values of $p=0.006$ and $p=0.03$, respectively. b Percentages of p53 immunopositivity determined by immunohistochemistry in different subgroups of IBD patients. Biopsy specimens of four follow-up visits of the cases (ND $n=3$, CRC $n=1$ ) and ten follow-up visits of controls (ND $n=9$, IND $n=1$ ) could not be graded due to

\subsection{Prognostic value of the biomarkers}

To compare the prognostic value of an abnormal DNA content, p53 immunopositivity and histological activity versus LGD for predicting neoplastic progression in subgroups of IBD patients, Cox regression with time dependent covariates severity of inflammation or a limited amount of tissue. p53 overexpression is defined as moderate and intense brown staining in $>15 \%$ of the nuclei. $P$-value from random effect regression analyses for difference between the cases and controls in p53 immunopositivity is $p=0.5$. In the ND-group the $p$-value for $\mathrm{p} 53$ immunopositivity was $p=0.6$. c Percentages of abnormal DNA content and p53 immunopositivity in different subgroups of IBD patients. $P$-values from random effect regression analyses for difference between the cases and controls in abnormal DNA content and p53 immunopositivity is $p=0.3$ for DI $0.96-1.05 / \mathrm{p} 53+, p=0.1$ for DI $1.06-1.34 / \mathrm{p} 53-, p=0.1$ for DI $1.06-1.34 / \mathrm{p} 53+, p=0.04$ for DI $>1.34 / \mathrm{p} 53-$ ,$p=1.0$ for $\mathrm{DI}>1.34 / \mathrm{p} 53+$. In the ND-group these $p$-values were $p=0.4$, $p=0.2, p=0.2, p=0.03, p=1.0$ respectively. DI $0.96-1.05$ and $p 53$ - have been used as reference conditions. $N D$, no dysplasia; $L G D$, low grade dysplasia; $H G D$, high grade dysplasia; $C R C$, colorectal cancer

was performed. With adjustment for age, significant associations with progression to advanced neoplasia were found for LGD, abnormal DNA ploidy, p53 immunopositivity, and moderate and severe colitis (Table 2).

Considering DNA ploidy with a DI 1.06-1.34 (neardiploid) over the length of surveillance as positive, a 4.7- 
a

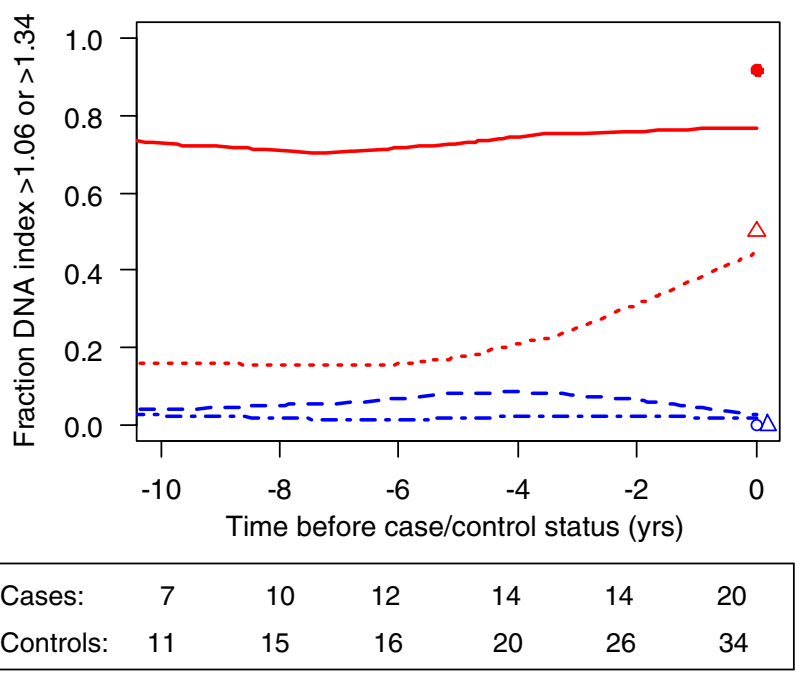

b

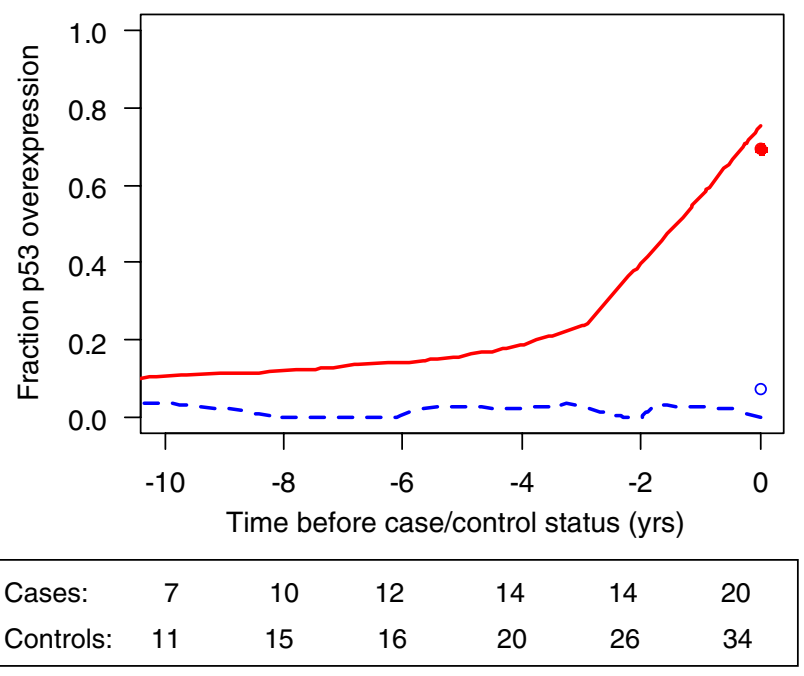

Fig. 3 Fraction of biopsy specimens with abnormal DNA content/p53 immunopositivity over time prior to the development of colorectal cancer $(\mathrm{CRC})$. a Fraction of abnormal DNA content._- $=$ cases, DI $\geq 1.06 ; \ldots \ldots . .=$ cases, DI $>1.34 ;-\boldsymbol{-}=$ controls, DI $\geq 1.06$ and $\boldsymbol{-} \cdot \mathbf{-}=$ controls, DI $>1.34$. b Fraction of p53 overexpression. $\longrightarrow=$ cases $;-\mathbf{Z}-\mathbf{Z}=$ controls. The numbers under each figure represent the number of IBD patients at that time point. The symbols at time point zero represent the fraction of patients at either time point with progression (cases $n=20$ ) or at last follow-up visit without progression (controls $n=34$ )

fold increased risk for neoplastic progression was calculated. When DNA ploidy marked as DI $>1.34$ (aneuploid) was used as criterion, the risk of advanced neoplasia was 6.6-fold increased. For p53 immunopositivity, a 3.0-fold increased risk for advanced neoplasia was observed over time. For mild, moderate and severe colitis, the age adjusted analyses revealed respectively a 2.3, 4.1, and 3.4-fold increased risk for advanced neoplasia. For LGD, the risk for neoplastic progression was established at 5.5-fold.
When the risk values were adjusted for both age and presence of LGD, abnormal DNA ploidy, p53 immunopositivity, and moderate and severe colitis remained statistically significant predictive of neoplasia (Table 2). The risks for advanced neoplasia were established at 3.6-fold for a DI 1.06-1.34, 5.3-fold for a DI >1.34, 1.7-fold for p53 immunopositivity, and 4.0 and 4.7-fold for moderate and severe colitis.

Adjustment for all confounders revealed significant relationships between DNA ploidy status, p53 immunopositivity and moderate colitis, and the risk for later development of advanced neoplasia (Table 2). The increased risks for advanced neoplasia was calculated at 4.3 -fold for a DI 1.06 1.34, and 4.8-fold for a DI $>1.34$. For p53 immunopositivity and moderate colitis, the risk was established at 1.7 and 3.0fold, respectively.

\section{Discussion}

Many centers perform colonoscopic surveillance in patients with longstanding IBD, but despite these programs, CRC remains an important cause of IBD-related mortality [20]. Particularly for patients with PSC and/or extensive colitis with a duration of $>8$ years, conventional colonoscopic surveillance with random biopsy sampling every 1-3 year seems to be insufficient [6, 23, 30]. Several recent studies have focused on the value of adding specific biomarkers to predict the development of IBDrelated CRC [16, 22, 25, 34].

At present, DNA ploidy and p53 are recognized as promising candidates for prediction of IBD-related CRC. Their obvious advantage is that they are both diffusely expressed and present at an early stage in the IBD related dysplasia-carcinoma sequence (Fig. 2, and ref. [17, 18, 22, 26]). However, the relative risk of abnormal DNA ploidy and p53 immunopositivity in developing IBD-related CRC has never been calculated. In the present study, a casecontrol study design based on our IBD surveillance database was performed to determine the risk of advanced neoplasia of both markers in subgroups of IBD patients. Potential biases in the study population were avoided by matching for age, gender, colitis characteristics, age of onset, duration of the disease, interval of follow-up, presence of PSC, and previous surgery.

In our study population, in age adjusted analyses significant relationships were observed between development of advanced neoplasia and prior presence of LGD (HR5.5), abnormal DNA ploidy (DI 1.06-1.34, HR4.7, and DI $>1.34$, HR6.6), and p53 immunopositivity (HR3.0) over time. When histological results were taken into account, i.e. the presence of LGD, both DNA ploidy and p53 immunopositivity maintained statistical significant predictors for 
Table 2 Prognostic value of LGD, abnormal DNA ploidy, p53 immunopositivity and inflammatory activity for neoplastic progression in highrisk IBD patients according to Cox regression analysis with time-dependent covariates

\begin{tabular}{|c|c|c|c|c|c|c|}
\hline \multirow[t]{2}{*}{ Variable } & \multicolumn{2}{|c|}{ Adjusted for age } & \multicolumn{2}{|c|}{ Adjusted for age and LGD } & \multicolumn{2}{|c|}{ Adjusted for all other variables } \\
\hline & HR $(95 \% \mathrm{CI})$ & $p$ & HR $(95 \% \mathrm{CI})$ & $p$ & HR $(95 \% \mathrm{CI})$ & $p$ \\
\hline LGD & $5.5(2.6-11.5)$ & $<0.0001$ & & & $2.0(0.8-4.9)$ & 0.2 \\
\hline DNA ploidy, $1.06 \leq \mathrm{DI} \leq 1.34^{\mathrm{a}}$ & $4.7(2.9-7.8)$ & $<0.0001$ & $3.6(2.2-5.9)$ & $<0.0001$ & $4.3(2.5-7.2)$ & $<0.0001$ \\
\hline DNA ploidy, DI $>1.34$ & $6.6(3.7-11.7)$ & $<0.0001$ & $5.3(3.3-8.3)$ & $<0.0001$ & $4.8(2.3-9.6)$ & $<0.0001$ \\
\hline p53 immunopositivity ${ }^{\mathrm{b}}$ & $3.0(1.9-4.7)$ & $<0.0001$ & $2.2(1.3-3.7)$ & 0.002 & $1.7(1.0-3.1)$ & 0.04 \\
\hline Mildly active inflammation & $2.3(0.9-5.3)$ & 0.07 & $2.1(0.8-5.4)$ & 0.1 & $1.9(0.7-4.9)$ & 0.2 \\
\hline Moderately active inflammation & $4.1(1.8-9.1)$ & 0.001 & $4.0(1.7-9.6)$ & 0.002 & $3.0(1.2-7.2)$ & 0.02 \\
\hline Severely active inflammation & $3.4(1.4-8.2)$ & 0.005 & $4.7(2.2-9.9)$ & $<0.0001$ & $2.3(0.9-5.9)$ & 0.1 \\
\hline
\end{tabular}

${ }^{a}$ DI, DNA index, calculated as ratio of the abnormal $G_{0} / G_{1}$ mean peak channel number to the normal diploid $G_{0} / G_{1}$ mean peak channel number

${ }^{\mathrm{b}}$ p53 immunopositivity, defined as moderate and intense brown staining in $>15 \%$ of the nuclei [14]

$L G D$, low grade dysplasia

development of advanced neoplasia (Table 2). Moreover, after adjustment for more confounders, abnormal DNA ploidy and p53 immunopositivity remained statistically significant predictive of neoplasia, with increasing risk for abnormal DNA ploidy depending on higher DI levels (Table 2).

Although risk stratification based on severity of histological signs of inflammation in IBD-related CRC was not the primary goal of this paper, we observed a correlation between severity of histological inflammation and increasing risk of neoplasia (Table 2). Evaluation of surveillance biopsy specimens showed that cases more often had histological signs of moderate colitis compared to the controls, in the years prior to the development of advanced neoplasia (HR 3.0). This observation is in agreement with recently published data [13,31], and supports the concept that severity of inflammation in IBD patients is a risk factor for IBD-related neoplasia [13].

Despite the advantage of our case-control study design, there are several potential limitations to this report. Firstly, all patient material was tested retrospectively. Unfortunately, a prospective trial analogous to our study will encounter large difficulties and costs because of required patient numbers, years of follow-up, and surveillance colonoscopies needed $[6,7]$. Secondly, our study was based on immunohistochemistry, which has the intrinsic shortcoming that the amount of staining is not linearly related to the amount of protein present, and may vary with the kit used.

Albeit most colonoscopies were performed during remission of the disease, some biopsy specimens have been taken when remission could not be achieved. The presence of histological signs of inflammation, and the regional or patchy presence of dysplasia, abnormal DNA ploidy and p53 immunopositivity $[16,21]$ which may even change over time $[3,24]$, might be a third putative drawback of the study. However, we tried to minimize these problems by using multiple biopsy specimens taken from different locations of the colon, validated scoring systems and two experts to evaluate histology and p53 immunopositivity. In order to calculate the hazard ratios we had to assume that the biopsy results at each location and time point accurately represented the grade of neoplasia, DNA ploidy status and p53 immunoreactivity in the colon at that interval. Obviously, this may not be correct, but given the number of specimens tested per patient, we expect that this bias was minimal.

Although we studied patients with UC as well as CD, no subdivision was made between the patient groups. This decision to interpret all results in the same analysis was based on the finding that no clear differences were seen between histology, DNA ploidy status and p53 immunoreactivity within the two different patient groups (data not shown).

Cox regression analysis with time-dependent covariates and random effect regression analyses in the ND-group revealed that patients positive for DNA ploidy and/or p53 are at high-risk of developing CRC. Therefore, extending the conventional colonoscopic surveillance with DNA ploidy and p53 could permit assigning IBD patients to either a low-risk (negative for dysplasia, DNA ploidy and p53), intermediate-risk (positive for dysplasia, DNA ploidy or p53) or high-risk (positive for dysplasia and DNA ploidy/p53) group. This refinement of risk stratification is of interest, since it would allow to allocate the resources more appropriate by reducing the number of unnecessary endoscopies for patients at a low-risk of developing CRC, while enabling more intensive surveillance endosocopies for those at high-risk of developing CRC. The results of this study suggest that the interval of colonoscopic surveillance in low-risk patients can be deferred up to 
5 years. This has to be evaluated in future prospective studies. The surveillance in intermediate- and high-risk patients should however be intensified to every year and every 3 months, respectively. In the patients at risk, novel endoscopy techniques, such as high magnification endoscopy, chromoendoscopy, narrow band imaging, or autofluorescence endoscopy may enhance the detection of suspicious lesions $[12,27,28,35]$.

In conclusion, the data from our study support the notion that the use of DNA ploidy and p53 enhance the sensitivity of correctly selecting patients at an increased risk of IBD-related CRC development. Given the retrospective nature of our study and its limited power, the potential utility of DNA ploidy and p53 immunohistochemistry into clinical management of patients with longstanding IBD warrants further investigation.

Acknowledgement This project was financially supported by the Erasmus MC by a grant to M.M. Gerrits (Mrace - Doelmatigheid \& Zorg; project no. DRP/REJ/271190).

Open Access This article is distributed under the terms of the Creative Commons Attribution Noncommercial License which permits any noncommercial use, distribution, and reproduction in any medium, provided the original author(s) and source are credited.

\section{References}

1. A. Ahmadi, S. Polyak, P.V. Draganov, Colorectal cancer surveillance in inflammatory bowel disease: the search continues. World J. Gastroenterol. 15, 61-66 (2009)

2. F.A. Farraye, R.D. Odze, J. Eaden, S.H. Itzkowitz. AGA medical position statement on the diagnosis and management of colorectal neoplasia in inflammatory bowel disease. Gastroenterology $\mathbf{1 3 8}$, 738-745 (2010)

3. R. Befrits, C. Hammarberg, C. Rubio, E. Jaramillo, B. Tribukait, DNA aneuploidy and histologic dysplasia in long-standing ulcerative colitis. A 10-year follow-up study. Dis. Colon Rectum 37, 313-319 (1994). discussion 9-20

4. S.R. Cairns, J.H. Scholefield, R.J. Steele, M.G. Dunlop, H.J. Thomas, G.D. Evans, J.A. Eaden, M.D. Rutter, W.P. Atkin, B. P. Saunders, A. Lucassen, P. Jenkins, P.D. Fairclough, C.R. Woodhouse, Guidelines for colorectal cancer screening and surveillance in moderate and high risk groups (update from 2002). Gut 59, 666-689 (2010)

5. M.M. Claessen, M.E. Schipper, B. Oldenburg, P.D. Siersema, G.J. Offerhaus, F.P. Vleggaar, WNT-pathway activation in IBDassociated colorectal carcinogenesis: potential biomarkers for colonic surveillance. Cell. Oncol. 32, 303-310 (2010)

6. P.D. Collins, C. Mpofu, A.J. Watson, J.M. Rhodes, Strategies for detecting colon cancer and/or dysplasia in patients with inflammatory bowel disease. Cochrane Database Syst. Rev. (2006):CD000279

7. J. Eaden, Review article: colorectal carcinoma and inflammatory bowel disease. Aliment. Pharmacol. Ther. 20, 24-30 (2004)

8. J.A. Eaden, J.F. Mayberry, Guidelines for screening and surveillance of asymptomatic colorectal cancer in patients with inflammatory bowel disease. Gut 51, V10-V12 (2002)

9. J. Eaden, K. Abrams, H. McKay, H. Denley, J. Mayberry, Interobserver variation between general and specialist gastrointestinal pathologists when grading dysplasia in ulcerative colitis. J. Pathol. 194, 152-157 (2001)

10. K. Geboes, R. Riddell, A. Ost, B. Jensfelt, T. Persson, R. Lofberg, A reproducible grading scale for histological assessment of inflammation in ulcerative colitis. Gut 47, 404-409 (2000)

11. W. Giaretti, S. Molinu, J. Ceccarelli, C. Prevosto, Chromosomal instability, aneuploidy, and gene mutations in human sporadic colorectal adenomas. Cell. Oncol. 26, 301-305 (2004)

12. M. Goetz, M.F. Neurath, Imaging techniques in inflammatory bowel disease: recent trends, questions and answers. Gastroentérol. Clin. Biol. 33, S174-S182 (2009)

13. R.B. Gupta, N. Harpaz, S. Itzkowitz, S. Hossain, S. Matula, A. Kornbluth, C. Bodian, T. Ullman, Histologic inflammation is a risk factor for progression to colorectal neoplasia in ulcerative colitis: a cohort study. Gastroenterology 133, 1099-1105 (2007). quiz 340-1

14. M. Hage, P.D. Siersema, K.J. Vissers, E.W. Steyerberg, J. Haringsma, E.J. Kuipers, H. van Dekken, Molecular evaluation of ablative therapy of Barrett's oesophagus. J. Pathol. 205, 57-64 (2005)

15. D.W. Hedley, M.L. Friedlander, I.W. Taylor, C.A. Rugg, E.A. Musgrove, Method for analysis of cellular DNA content of paraffin-embedded pathological material using flow cytometry. J. Histochem. Cytochem. 31, 1333-1335 (1983)

16. K. Holzmann, M. Weis-Klemm, B. Klump, C.J. Hsieh, F. Borchard, M. Gregor, R. Porschen, Comparison of flow cytometry and histology with mutational screening for p53 and Ki-ras mutations in surveillance of patients with long-standing ulcerative colitis. Scand. J. Gastroenterol. 36, 1320-1326 (2001)

17. S.P. Hussain, P. Amstad, K. Raja, S. Ambs, M. Nagashima, W.P. Bennett, P.G. Shields, A.J. Ham, J.A. Swenberg, A.J. Marrogi, C. C. Harris, Increased p53 mutation load in noncancerous colon tissue from ulcerative colitis: a cancer-prone chronic inflammatory disease. Cancer Res. 60, 3333-3337 (2000)

18. S. Itzkowitz, Colon carcinogenesis in inflammatory bowel disease: applying molecular genetics to clinical practice. J. Clin. Gastroenterol. 36, S70-S74 (2003). discussion S94-6

19. S.H. Itzkowitz, N. Harpaz, Diagnosis and management of dysplasia in patients with inflammatory bowel diseases. Gastroenterology 126, 1634-1648 (2004)

20. P. Karlen, R. Lofberg, O. Brostrom, C.E. Leijonmarck, G. Hellers, P.G. Persson, Increased risk of cancer in ulcerative colitis: a population-based cohort study. Am. J. Gastroenterol. 94, 10471052 (1999)

21. B. Klump, K. Holzmann, A. Kuhn, F. Borchard, M. Sarbia, M. Gregor, R. Porschen, Distribution of cell populations with DNA aneuploidy and p53 protein expression in ulcerative colitis. Eur. J. Gastroenterol. Hepatol. 9, 789-794 (1997)

22. B.A. Lashner, W.M. Bauer, L.A. Rybicki, J.R. Goldblum, Abnormal p53 immunohistochemistry is associated with an increased colorectal cancer-related mortality in patients with ulcerative colitis. Am. J. Gastroenterol. 98, 1423-1427 (2003)

23. C.H. Lim, M.F. Dixon, A. Vail, D. Forman, D.A. Lynch, A.T. Axon, Ten year follow up of ulcerative colitis patients with and without low grade dysplasia. Gut 52, 1127-1132 (2003)

24. R. Lofberg, O. Brostrom, P. Karlen, A. Ost, B. Tribukait, DNA aneuploidy in ulcerative colitis: reproducibility, topographic distribution, and relation to dysplasia. Gastroenterology 102, 1149-1154 (1992)

25. J.W. Nathanson, N.E. Yadron, J. Farnan, S. Kinnear, J. Hart, D.T. Rubin, p53 mutations are associated with dysplasia and progression of dysplasia in patients with Crohn's disease. Dig. Dis. Sci. 53, 474 $480(2008)$

26. C. Pohl, A. Hombach, W. Kruis, Chronic inflammatory bowel disease and cancer. Hepatogastroenterology 47, 57-70 (2000)

27. D. Ramsoekh, M.E. van Leerdam, M. van Ballegooijen, J.D. Habbema, E.J. Kuipers, Population screening for colorectal cancer: faeces, endoscopes or X-rays? Cell. Oncol. 29, 185-194 (2007) 
28. D. Ramsoekh, J. Haringsma, J. Poley, P. van Putten, H. van Dekken, E.W. Steyerberg, M.E. van Leerdam, E.J. Kuipers, A back-to-back comparison of white light video endoscopy with autofluorescence endoscopy for adenoma detection in high-risk subjects. Gut 59, 785-793 (2010)

29. R.H. Riddell, H. Goldman, D.F. Ransohoff, H.D. Appelman, C.M. Fenoglio, R.C. Haggitt, C. Ahren, P. Correa, S.R. Hamilton, B.C. Morson et al., Dysplasia in inflammatory bowel disease: standardized classification with provisional clinical applications. Hum. Pathol. 14, 931-968 (1983)

30. D.J. Robertson, E.R. Greenberg, M. Beach, R.S. Sandler, D. Ahnen, R.W. Haile, C.A. Burke, D.C. Snover, R.S. Bresalier, G. McKeown-Eyssen, J.S. Mandel, J.H. Bond, R.U. Van Stolk, R.W. Summers, R. Rothstein, T.R. Church, B.F. Cole, T. Byers, L. Mott, J.A. Baron, Colorectal cancer in patients under close colonoscopic surveillance. Gastroenterology 129, 34-41 (2005)
31. M. Rutter, B. Saunders, K. Wilkinson, S. Rumbles, G. Schofield, M. Kamm, C. Williams, A. Price, I. Talbot, A. Forbes, Severity of inflammation is a risk factor for colorectal neoplasia in ulcerative colitis. Gastroenterology 126, 451-459 (2004)

32. T.V. Shankey, P.S. Rabinovitch, B. Bagwell, K.D. Bauer, R.E. Duque, D.W. Hedley, B.H. Mayall, L. Wheeless, C. Cox, Guidelines for implementation of clinical DNA cytometry. International Society for Analytical Cytology. Cytometry 14, 472-477 (1993)

33. U. Sjoqvist, Dysplasia in ulcerative colitis-clinical consequences? Langenbecks Arch. Surg. 389, 354-360 (2004)

34. U. Sjoqvist, R. Befrits, S. Soderlund, A. Ost, P. Karlen, B. Tribukait, C. Rubio, P. Rutgeerts, K. Geboes, R. Lofberg, Colorectal cancer in colonic Crohn's disease-high frequency of DNA-aneuploidy. Anticancer Res. 25, 4393-4397 (2005)

35. A. Stallmach, C. Bielecki, C. Schmidt, Malignant transformation in inflammatory bowel disease - surveillance guide. Dig. Dis. 27, 584-590 (2009) 